\title{
Effects of solution viscosity on perceived saltiness and sweetness
}

\author{
CAROL M. CHRISTENSEN \\ Monell Chemical Senses Center, Philadelphia, Pennsylvania 19104
}

\begin{abstract}
Subjects used magnitude estimation to judge the perceived saitiness or sweetness of a series of aqueous solutions containing five suprathreshold concentrations of $\mathrm{NaCl}$ or sucrose and thickened with sodium carboxymethylcellulose (CMC). In the first experiment, CMC-H (high viscosity form) was used to thicken a series of sucrose and $\mathrm{NaCl}$ solutions to six viscosity levels (1-2,025 centistokes). At the highest viscosity levels, significant decreases occurred in the perceived taste intensity of only the lower concentrations of sucrose and $\mathrm{NaCl}$. A second experiment determined that variations in the quantity of solution sampled from cups did not systematically influence judgments of saltiness when the starting volume was $10 \mathrm{ml}$. In the third experiment, aqueous solutions containing sucrose or $\mathrm{NaCl}$ were thickened with the low (L), medium (M), or high (H) viscosity form of CMC (1-1,296 centistokes). CMC-Lthickened solutions produced little or no suppression of perceived taste intensity, whereas viscous CMC-H solutions produced significant reductions in perceived saltiness and sweetness.
\end{abstract}

The starches and gums, high molecular weight polysaccharides, are ubiquitous food additives. Their addition can have the effect of thickening, which is often the reason for their use. A possible consequence of the thickening produced by gums and starches is that taste perception could be altered.

There are several sites where changes in solution viscosity could affect perceived taste intensity. Physicalchemical interactions between the thickener and taste stimuli in the oral cavity could, among other possibilities, change the diffusion rate or accessibility of taste substances to the taste receptor surface. Interactions could also be occurring in the nervous system where trigeminal and gustatory inputs converge or at a perceptual level where dietary experiences may differentially influence taste judgments in thick and thin solutions (Christensen, 1980).

Previous research (see review by Pangborn, Trabue, \& Szczesniak, 1973) suggests that the addition of gums or starches to a liquid medium frequently raises taste thresholds and reduces the perceived intensity of suprathreshold concentrations of taste substances. Only a few studies have systematically varied gum concentrations (viscosity levels) in order to evaluate the relationships between solution viscosity and perceived taste intensity. In one pair of studies (Pangborn

This research was supported in part by NIH Training Grant 5T32NS-07068 from NINCDS to the Monell Chemical Senses Center and a Grant-in-Aid from Sigma Delta Epsilon to the author. Appreciation is expressed to the Hercules Corporation, Wilmington, Delaware, particularly to Hal Jabloner, for supplying the CMC and for providing valuable advice. The author also wishes to thank Gary Beauchamp and Robert Cagan of the Monell Center for their helpful comments and Davis Diehl for her technical support.
\& Szczesniak, 1974; Pangborn, Trabue, \& Szczesniak, 1973), perceived taste or flavor intensity was measured in aqueous solutions thickened with several concentrations of five different gums. The data did not reveal a general effect of viscosity on taste or flavor intensity. Instead, the changes in taste or flavor intensity observed could best be attributed to a specific interaction between a single gum and a single taste or flavor substance. This change was frequently independent of differences in solution viscosity. However, the generality of these results is limited by the narrow range of viscosities utilized by the researchers [1 to 72 centipoises (cps)].

In another series of studies, magnitude estimates were obtained of the taste intensity of six suprathreshold concentrations of glucose, citric acid, quinine sulfate, and sodium chloride dissolved in aqueous solutions thickened with sodium carboxymethylcellulose (CMC) from 1 to 1,000 cps (Moskowitz \& Arabie, 1970). Perceived taste intensity decreased as a function of viscosity for all the tested taste stimuli. Power functions described the relationships between solution viscosity levels and perceived taste intensity giving exponent values ranging from $-.06(\mathrm{NaCl})$ to -.18 (quinine). In a similarly designed study, Arabie and Moskowitz (1971) extended the viscosity range to $10,000 \mathrm{cps}$ and investigated the effects of viscosity on the perceived sweetness of solutions containing sucrose or saccharin. Perceived sweetness decreased as a function of solution viscosity, with the average power function exponent being approximately -.20 for the two taste substances.

The results of the two sets of studies lead to opposite conclusions; whether solution viscosity has a pervasive or selective effect on perceived taste 
intensity remains to be determined. The major objective of the present research was to better define the effects of solution viscosity on taste perception by incorporating a number of procedural controls which facilitated interpretation of the results. These included careful selection and control of physical viscosity levels, separation and evaluation of changes in taste perception due to changes in solution viscosity per se rather than to other concentration-related properties accompanying the addition of gums or starches, and evaluation of the contribution of standard testing procedures to possible artifactual decrements in perceived taste intensity.

\section{EXPERIMENT 1}

The high-viscosity form of sodium carboxymethylcellulose (CMC-H) is a thickening agent that has been used in successful demonstrations of decreases in perceived taste intensity with increasing solution viscosity (e.g., Moskowitz \& Arabie, 1970). The first experiment examined this effect of $\mathrm{CMC}$ after several procedural controls had been added.

Viscosities of CMC-thickened solutions are changed upon the addition of most taste stimuli. In previous studies, solution viscosities were variable because fixed quantities of thickeners were added to taste solutions. In the present study, viscosity levels were held constant by separately determining for every taste substance and concentration the amount of CMC required to thicken aqueous solutions to a particular level. Also, because CMC-thickened solutions are non-Newtonian and, thus, the apparent viscosities of these solutions are dependent on their shear rate, solution viscosities were determined at shear rates and temperatures hypothesized to exist in the oral cavity (Shama \& Sherman, 1973).

Changes were also made in the solution presentation method. Typically, subjects are given a measured quantity of each sample solution in small cups. This procedure results in residual amounts of the thicker solutions remaining in the test cups (see Experiment 2). To insure that subjects sampled equal quantities of thick and thin solutions, a syringe fitted with a plastic straw was used to present each solution.

The effects of variously thickened $\mathrm{CMC}-\mathrm{H}$ solutions on perceived saltiness and sweetness were measured by using the method of magnitude estimation. Five suprathreshold concentrations each of sucrose and sodium chloride were chosen to represent the intensity spectrum from weak to strong tasting, and these solutions were thickened to six physical viscosity levels to represent a broad range of viscosities [12,025 centistokes (csk)].

\section{Method}

Subjects. Ten university students served as subjects. They were solicited by advertisements displayed on local campuses. Respondents were chosen in order of inquiry, with the restriction that there be equal numbers of males and females. The subjects were paid for their participation.

Preparation of test stimuli. Ten different taste solutions containing either sodium chloride or sucrose were prepared with deionized water. The solutions were $.05, .10, .20, .40$, and $.80 \mathrm{M}$ sodium chloride $(\mathrm{NaCl})$ and $.06, .12, .25, .50$, and $1.00 \mathrm{M}$ sucrose. Each of the taste solutions was thickened to $100,300,900,1,350$, and 2,025 csk with the high viscosity form of sodium carboxymethylcellulose (CMC-H, Hercules, Inc., Wilmington, Del.). Methods for the preparation and measurement of viscous solutions have been previously described (Christensen, 1980).

Measurement of perceived taste intensity. The subjects used the method of magnitude estimation to report perceived taste intensity. Prior to judging the test solutions, the subjects were given brief training in the use of magnitude estimation by being asked to judge the circumference of a series of circles.

The test solutions were presented in random order to subjects, but the first sample was either an unthickened ( $1 \mathrm{csk}) .06 \mathrm{M}$ sucrose solution or a $.05 \mathrm{M} \mathrm{NaCl}$ solution. No numerical value was assigned by the experimenter to the first sample of the session (modulus-free design). The subjects were not permitted to use negative numbers but could use zero or positive whole numbers, decimals, and fractions to express the perceived saltiness or sweetness of the solutions.

Testing procedures. The subjects participated in two daily test sessions, each lasting approximately $1 \mathrm{~h}$. Only one taste quality was tested during a single session. The order of presentation of the taste qualities was randomized for each subject. The 30 different solutions [ 5 taste concentrations $\times 6$ viscosity levels (includes unthickened level-1 csk)] were presented twice to subjects in a given test session.

The subjects sampled $10 \mathrm{ml}$ of each solution from a plastic disposable syringe fitted with an 8-cm piece of Tygon tubing that served as a straw. The Tygon tubing protruded through a slot in an opaque barrier that separated the experimenter and the subject. Efforts were made to hold constant the rate of solution delivery.

The subjects were instructed to swish the test solution around in their mouths as they would a mouthwash, expectorate, and then report its perceived saltiness or sweetness. Two deionized water rinses preceded the presentation of the next test solution.

Analysis. A few subjects reported zero scores. In order to analyze the data, the zero scores were converted to half the value of the lowest positive score reported by that subject. The raw data were normalized between subjects by using the mean of the replicate judgments. Normalization was accomplished by obtaining the differences between the $\log$ grand mean and the log means of individual subjects and adding this value to each subject's set of log judgments (Engen, 1971). Appropriate power functions were calculated from the normalized data.

Two-factor repeated measures analyses of variance (Taste Concentration by Viscosity Level) were performed on the log data. Separate analyses were performed for the sucrose and $\mathrm{NaCl}$ judgments. For selected post hoc comparisons, $t$ tests were used, and the significance level $(.05)$ was adjusted for multiple comparisons by dividing it by the number of tests performed (Winer, 1971).

\section{Results}

The addition of CMC-H to aqueous solutions containing $\mathrm{NaCl}$ or sucrose produced a significant reduction in perceived taste intensity. Though the main effect of viscosity level was significant $[F(5,45)=5.56$ for sucrose and 3.57 for $\mathrm{NaCl}, \mathrm{p}<.01$ ], the interaction between taste concentration and viscosity level was also significant $[F(20,180)=1.99$ for sucrose and 2.75 for $\mathrm{NaCl}, \mathrm{p}<.01]$. The interaction is illustrated in Figures 1 and 2; increases in solution viscosity led to decreases in the perceived taste intensity of only 


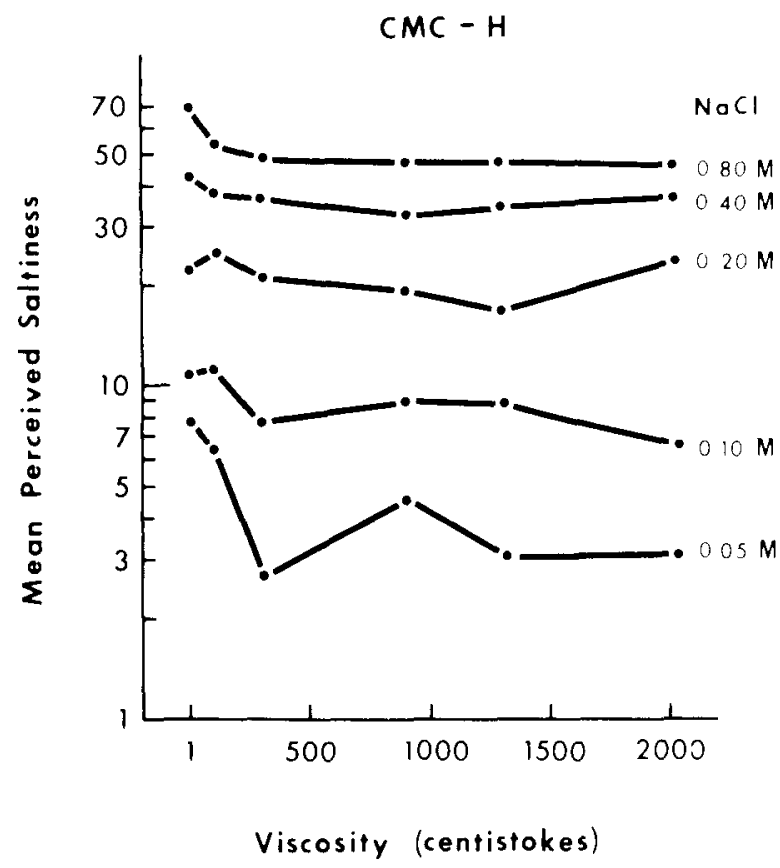

Figure 1. Effect of CMC-H-thickened solutions on perceived sweetness at five sucrose concentrations. Each point is the geometric mean for 10 subjects.

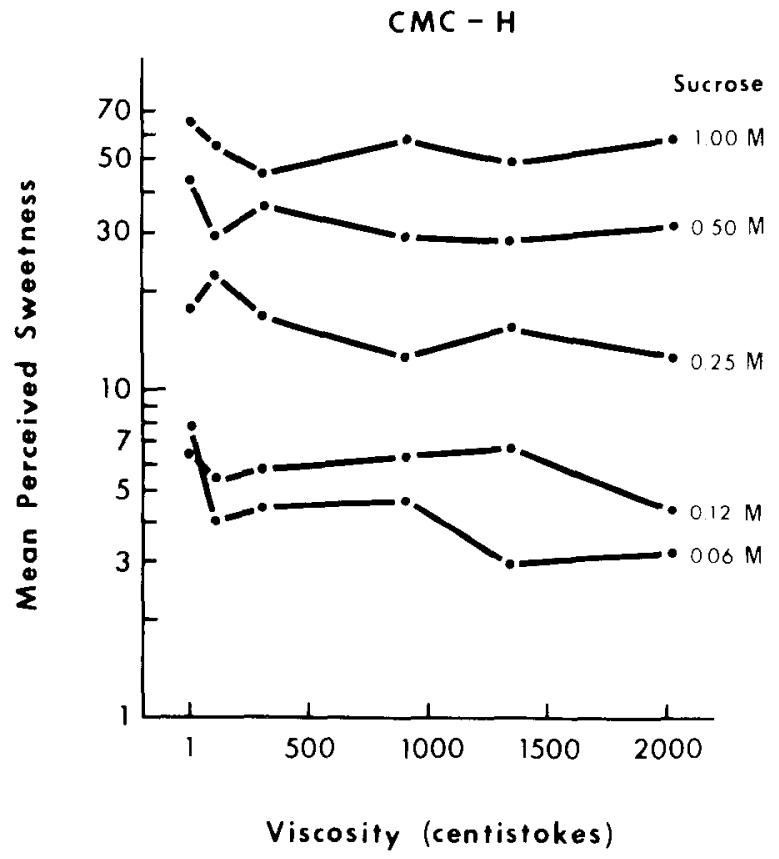

Figure 2. Effect of CMC-H-thickened solutions on perceived saltiness at five $\mathrm{NaCl}$ concentrations. Each point is the geometric mean for 10 subjects.

the lower concentrations of sucrose and $\mathrm{NaCl}$. The perception of the intensity of the higher taste concentrations was largely unaffected by increasing additions of CMC-H.

Perceived taste intensities of sucrose and $\mathrm{NaCl}$ solutions were affected similarly by increasing solution viscosity (Figures 1 and 2). For the lowest taste concentrations $(.06 \mathrm{M}$ sucrose and .05 M NaCl), post hoc tests revealed that the unthickened solutions ( 1 csk) were perceived to be significantly stronger tasting than any of the thickened solutions. For solutions containing either $.12 \mathrm{M}$ sucrose or $.10 \mathrm{M} \mathrm{NaCl}$, the unthickened solutions were perceived to be significantly stronger tasting than only the thickest solutions $(2,025 \mathrm{csk})$. No other significant differences were observed between any of the solutions.

Power functions were calculated to describe perceived taste intensity as a function of solution viscosity at each taste concentration level. The exponents ranged from $-.14\left(r^{2}=.20\right)$ for the $.06 \mathrm{M}$ sucrose solutions to -.02 for the $1.00 \mathrm{M}$ solutions and from $-.17\left(\mathrm{r}^{2}=.22\right)$ for the $.05 \mathrm{M} \mathrm{NaCl}$ solutions to +.04 for the $.80 \mathrm{M}$ solutions.

\section{Discussion}

This study establishes that the addition of $\mathrm{CMC}-\mathrm{H}$ to aqueous solutions does affect perceived saltiness and sweetness, but that the effects are observed only with weak-tasting solutions. The results were dramatic; there was approximately a $60 \%$ reduction in perceived taste intensity when either a $.06 \mathrm{M}$ sucrose or a $.05 \mathrm{M}$ $\mathrm{NaCl}$ solution was thickened to 2,025 csk.

Pangborn et al. (1973) did not report taste concentration-related effects in their experiment, nor did Arabie and Moskowitz (1971) or Moskowitz and Arabie (1970). However, in the latter papers, there was evidence that such an effect was present. Lower concentrations of several of the taste substances were most affected by the addition of the gum, CMC.

The exponents for the power functions relating perceived taste intensity as a function of solution viscosity at each taste concentration level were comparable to those found in previous research with $\mathrm{NaCl}$ solutions (Moskowitz \& Arabie, 1970) but lower than those found with sucrose solutions (Arabie \& Moskowitz, 1971). However, the viscosity range extended to $10,000 \mathrm{cps}$ in the latter study. As evidenced by the low $r^{2}$ values and as illustrated in Figures 1 and 2, power functions as well as other linear functions do not adequately represent the data.

\section{EXPERIMENT 2}

Differences in the quantity of solution sampled by subjects might be partly responsible for the published observations of a depression in taste intensity with increasing solution viscosity. Pilot studies demonstrated that the standard testing procedure of presenting solutions to subjects in small cups resulted in residual amounts of the thicker solutions remaining in the test cups. Following the presentation of $10 \mathrm{ml}$ of variously thickened sucrose and $\mathrm{NaCl}$ solutions to 10 subjects, an average of $1 \mathrm{ml}$ of the 36 -csk solutions $(1.3 \pm .5 \mathrm{ml}), 2 \mathrm{ml}$ of the 216 -csk $(1.9 \pm .4 \mathrm{ml})$, and $3 \mathrm{ml}$ of the 1,296-csk solutions $(3.4 \pm .7 \mathrm{ml})$ remained in the test cups. The reductions in the quantities 
of the thicker solutions sampled by subjects could lead to a decrease in perceived taste intensity.

In this experiment, the subjects' judgments of the perceived saltiness of CMC-thickened solutions containing $\mathrm{NaCl}$ were compared using three methods of solution presentation. Ten-milliliter samples of solution were presented to subjects either in cups or syringes. Solutions were also presented in syringes in which the volume was reduced to correspond to amounts remaining in cups after sampling variously thickened solutions. The same subjects were used for all three presentation methods so that differences in judgments could not be attributed to sampling effects.

\section{Method}

Seven university students served as subjects. Methods for subject selection, preparation of test stimuli, and measurements of physical viscosity and perceived taste intensity were similar to those used in Experiment 1. Procedural differences are outlined below.

Test stimuli. A total of 30 different test solutions were prepared. Deionized water alone and deionized water containing one of five suprathreshold concentrations of $\mathrm{NaCl}(.05-.80 \mathrm{M})$ were thickened to 1 (unthickened), 6, 36, 216, and 1,296 csk with CMC-H.

Testing procedure. The subjects participated in three daily test sessions, each lasting approximately $1 \mathrm{~h}$. During each session, the subjects judged the saltiness of the 30 solutions, which were presented twice (a total of 60 solutions). All but the first solution were randomized for presentation during a single session; the first solution was from the middle taste concentration and viscosity ranges (.10 M NaCl at $216 \mathrm{csk})$.

Three different solution presentation methods were used, each on successive test days. For one method, $10 \mathrm{ml}$ of each test solution was presented in 30-ml plastic soufflé cups to the subjects. The other two delivery methods used the syringe system described in Experiment 1 , and the subjects received either a fixed quantity of solution $(10 \mathrm{ml})$ or a quantity of solution that was adjusted to correspond to the net volume sampled from the soufflé cups after residual amounts had been accounted for: $10 \mathrm{ml}$ of the 1 - and 6-csk solutions, $9 \mathrm{ml}$ of the 36 -csk solutions, $8 \mathrm{ml}$ of the 216 -csk solutions, and $7 \mathrm{ml}$ of the 1,296-csk solutions. The presentation order for the three methods was determined randomly for each subject.

Analysis. In order to compare magnitude estimates of saltiness obtained on successive days, each subject's judgments were first normalized between days by using the values obtained from the unthickened (1 csk) $\mathrm{NaCl}$ solutions. This set was chosen because it was assumed that the subjects' judgments of these solutions would have been unaffected by the stimulus presentation method and because these values were not included in the subsequent analysis of variance. Procedures for normalization were as described earlier (see Experiment 1).

\section{Results}

The solution presentation procedures had no major effect on the perception of the saltiness of variously thickened solutions. A three-factor analysis of variance (Delivery Procedure by Viscosity Level by Taste Concentration) performed on the normalized $\log$ data revealed no main effect attributable to the type of delivery system. The interaction between delivery procedures and viscosity level was significant $[F(6,36)$ $=2.90, \mathrm{p}<.05]$. The data are presented in Table 1 . Post hoc tests revealed that 6-csk solutions presented in $10-\mathrm{ml}$ quantities from a syringe were perceived as less salty than solutions presented by the other two methods (though no actual reduction in volume for the 6-csk solutions). At 1,296 csk, solutions presented in syringes in which the volume was reduced $(7 \mathrm{ml}$ for 1,296-csk solutions) were perceived to be significantly saltier than solutions presented by the other two methods.

As expected from Experiment 1, there was a significant interaction between taste concentration and viscosity level $[F(12,72)=5.86, p<.001]$. Though not illustrated here, only judgments of the lower concentrations of $\mathrm{NaCl}$ were affected by the addition of CMC-H.

\section{Discussion}

Solution delivery methods did not appear to contribute to the observed decreases in perceived taste intensity as a function of increasing $\mathrm{CMC}-\mathrm{H}$ concentration. Though there was a significant interaction between viscosity level and solution delivery method, post hoc tests revealed no meaningful pattern of the results. Variability in the data may have been responsible for the interaction's reaching statistical significance.

Judgments of perceived saltiness were unaffected by a $3-\mathrm{ml}$ reduction in solution volume when the starting volume was $10 \mathrm{ml}$. Similar reductions when the initial volume is less than $10 \mathrm{ml}$ may affect perceived taste intensity. In the series of studies reporting a pervasive effect of viscosity on perceived taste intensity, subjects were given either 3-5 ml volumes of test solution (Arabie \& Moskowitz, 1971) or 5-7 ml (Moskowitz \& Arabie, 1970).

\section{EXPERIMENT 3}

This experiment was designed to examine the nature of the observed effects on taste perception of the ad-

Table 1

Mean and Standard Error of Log Perceived Saltiness as a Function of Solution Delivery Method and Viscosity Level

Physical Viscosity

\begin{tabular}{|c|c|c|c|c|c|c|c|c|}
\hline \multirow[b]{2}{*}{ Delivery Method } & \multicolumn{2}{|c|}{$6 \mathrm{csk}$} & \multicolumn{2}{|c|}{$36 \mathrm{csk}$} & \multicolumn{2}{|c|}{$216 \mathrm{csk}$} & \multicolumn{2}{|c|}{$1,296 \mathrm{csk}$} \\
\hline & Mean & $\mathrm{SE}$ & Mean & $\mathrm{SE}$ & Mean & $\mathrm{SE}$ & Mean & $\mathrm{SE}$ \\
\hline Cups & 1.88 & .37 & 1.88 & .36 & 1.79 & .38 & 1.64 & .40 \\
\hline Syiinges, $10 \mathrm{ml}$ & 1.73 & .32 & 1.79 & .30 & 1.74 & .34 & 1.59 & .36 \\
\hline Syringes, $7-10 \mathrm{ml}$ & 1.99 & .33 & 1.88 & .36 & 1.79 & .37 & 1.77 & .36 \\
\hline
\end{tabular}

Note- $n=7$. Values obtained by collapsing over $\mathrm{NaCl}$ concentrations. 
dition of $\mathrm{CMC}-\mathrm{H}$ to aqueous solutions. Changes in solution viscosity may be responsible for decrements in taste intensity, but there are other physical and chemical properties of gums not being measured which covary with concentration and viscosity. These other variables may be responsible for the observed effects on taste perception. Therefore, in this study, three types of CMC [CMC-H (high), CMC-M (medium), and CMC-L (low)] were used; these do not differ in chemical properties but do differ in polymeric length and, hence, in their relative abilities to thicken solutions (Cellulose gum, 1971). Because approximately three times more $\mathrm{CMC}-\mathrm{L}$ than $\mathrm{CMC}-\mathrm{H}$ is required to thicken solutions to a particular viscosity level, it is possible to determine whether viscosity alone or other concentration-related effects, perhaps acting in concert with viscosity, are responsible for the observed changes in taste perception.

\section{Method}

Ten university students served as subjects in this experiment. Methods for the selection of subjects, preparation of test stimuli, measurements of physical viscosity and perceived taste intensity, and methods for data analysis were similar to those of Experiment 1. Procedural differences are outlined below.

Test stimuli. A total of 151 different test solutions were prepared. Deionized water alone and deionized water containing one of five suprathreshold concentrations of sucrose $(.06-1.00 \mathrm{M})$ or sodium chloride (.05-.80 M) were thickened to 1 (unthickened), 6 , 36,216 , and $1,296 \mathrm{csk}$. Pilot studies determined the quantities of CMC-L (7LF), CMC-M (7MF), and CMC-H (7H3SF) required to thicken each of the test solutions to the desired viscosity level. Viscosities were determined at $100 \mathrm{sec}^{-1}$ for the 6-csk solutions, at $40 \mathrm{sec}^{-1}$ for the 36-csk solutions, and at $10 \mathrm{sec}^{-1}$ for the 216and 1,296-csk solutions in order to comply with shear rates hypothesized to be operating in the oral cavity (Shama \& Sherman, 1973).

Testing procedures. The subjects participated in four daily test sessions, each lasting approximately $1 \mathrm{~h}$. A single taste quality was tested during a session. Replicate judgments were obtained, but, because of the large number of individual solutions ( 90 per session), replications were obtained on successive days. The presentation order for the taste qualities was balanced. All but the first solution was randomized for presentation during a single session. The first solution was chosen from the middle taste concentration and viscosity ranges and was either a $.25-\mathrm{M}$ sucrose or $.20-\mathrm{M} \mathrm{NaCl}$ solution thickened to $36 \mathrm{csk}$ with CMC-M.

\section{Results}

The results of this experiment demonstrated clearly that taste suppression did not necessarily occur following the addition of CMC to aqueous solutions. A summary of the data is illustrated in Figures 3 and 4. A four-factor analysis of variance (Taste Quality by Taste Concentration by Viscosity Level by CMCType) performed on the unnormalized log data revealed that the main effect of the type of CMC was significant $[F(2,18)=6.35, p<.01]$. Overall perceived taste intensity was higher in solutions thickened with CMC-L $(\log \bar{X}=1.19)$ than in solutions thickened with either CMC-M $(\log \overline{\mathrm{X}}=1.14)$ or CMC-H $(\log \overline{\mathrm{X}}$ $=1.14$ ). From an examination of Figures 3 and 4 , it

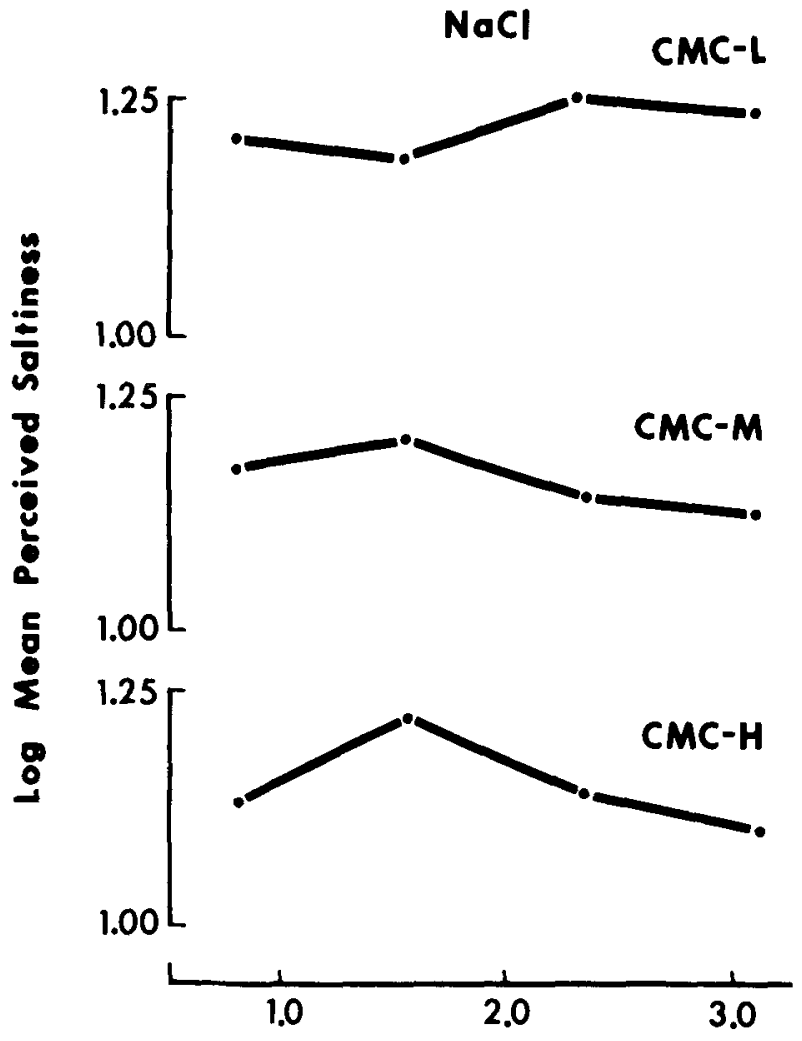

\section{Log Viscosity (centistokes)}

Figure 3. Effects of CMC-L-, CMC-M-, and CMC-H-thickened solutions on perceived sweetness. Each point is the log mean for 10 subjects for all five sucrose concentrations.

is evident that, unlike CMC-M or $\mathrm{CMC}-\mathrm{H}$, the addition of $\mathrm{CMC}-\mathrm{L}$ to solutions containing $\mathrm{NaCl}$ or sucrose did not appear to produce a depression in their perceived saltiness or sweetness.

The differential effectiveness of the three types of $\mathrm{CMC}$ in producing taste suppression was most obvious at higher viscosity levels [Viscosity Level by CMC-Type, $F(6,54)=2.15, p=.06]$. As confirmed by $t$ tests, solutions thickened with CMC-L were judged to have a significantly higher taste intensity than either CMC-M or CMC-H solutions when these solutions were thickened to 216 or $1,296 \mathrm{csk}$. There were no statistically significant differences between the three types of CMC when the solutions were prepared to a thickness of 6 or $36 \mathrm{csk}$.

The analysis of variance test found no significant main effect or significant interactions involving taste quality. As illustrated in Figures 3 and 4, the different types of CMC produced roughly similar patterns of results for both sucrose and $\mathrm{NaC} 1$ solutions.

If concentration-related properties of CMC are responsible for observed changes in perceived taste intensity, then there should be a correlation between perceived taste intensity and the amount of added CMC without regard to the type of CMC (low, med- 


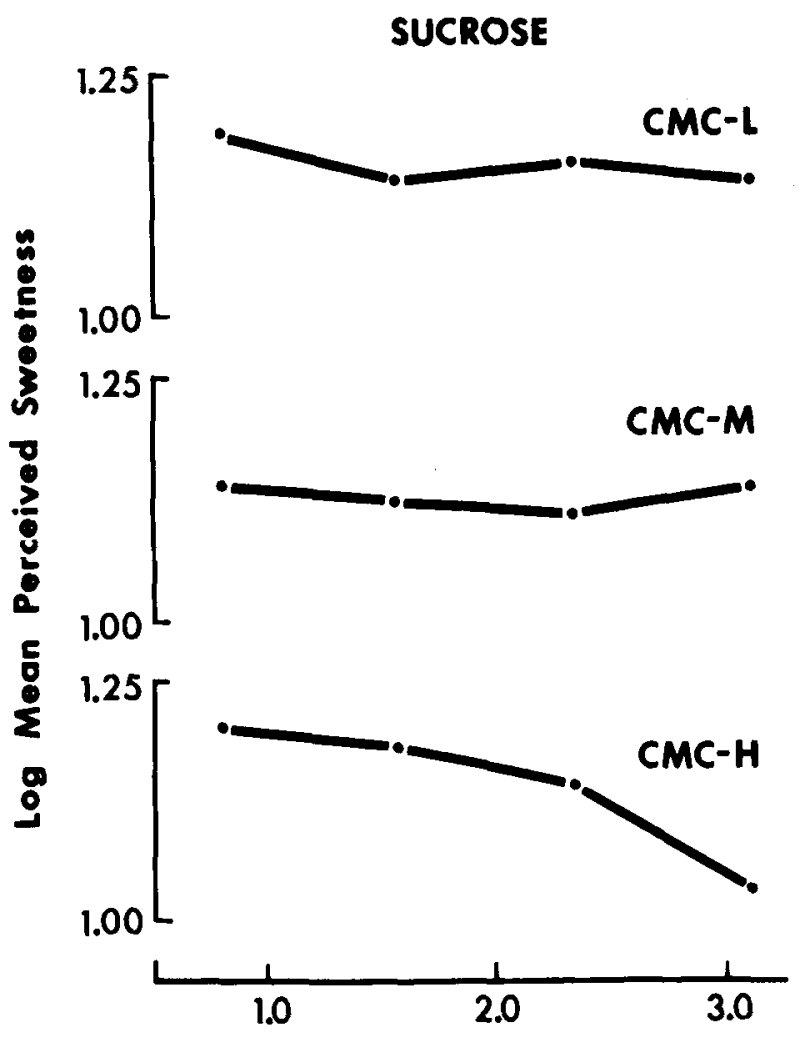

Log Viscosity (centistokes)

Figure 4. Effects of CMC-L-, CMC-M-, and CMC-H-thickened solutions on perceived saltiness. Each point is the $\log$ mean for 10 subjects for all five $\mathrm{NaCl}$ concentrations.

ium, or high viscosity types). The degree of linear correlation between the amount of CMC added to aqueous solutions and judgments of solution taste intensities were calculated from log transformed data. Correlations were computed separately for sucrose and $\mathrm{NaCl}$ solutions at each taste concentration level. The correlation coefficients were relatively low; the highest correlations were obtained at the lowest taste concentrations $(r=.54$ for $.05 \mathrm{M} \mathrm{NaCl}$ and $r=-.19$ for .06 sucrose solutions).

The three grades of CMC are reported to be odorless and tasteless (Cellulose gum, 1971). Subjects did not report significant increases in either perceived saltiness or sweetness following additions of CMC-L, $-\mathrm{M}$, or $-\mathrm{H}$ to deionized water. Median magnitude estimations of taste intensity were zero for unthickened and $\mathrm{CMC}$-thickened deionized water solutions containing no added taste substances. The single exception was a median magnitude estimate of 1.0 for the sweetness of deionized water thickened to $1,296 \mathrm{csk}$ with CMC-H.

\section{Discussion}

This experiment demonstrated that neither concentration-related properties nor viscosity-related properties of $\mathrm{CMC}$ were solely responsible for changes in taste perception. If the concentration of CMC produced the observed decreases in perceived taste intensity, then $\mathrm{CMC}-\mathrm{L}$ should have produced the greatest changes in taste perception because more of the low-viscosity type of CMC was required to achieve a particular viscosity level. Instead, the opposite results were observed. Furthermore, correlations between the quantities of added CMC and judgments of perceived taste intensity were low.

If changes in solution viscosity caused changes in taste perception, then there should be no significant differences among the gums. Yet there were significant differences; CMC-L produced little or no suppression of perceived taste intensity in thick or thin solutions, whereas $\mathrm{CMC}-\mathrm{H}$ produced significant reductions in perceived taste intensity.

\section{GENERAL DISCUSSION}

Two major findings emerge from this set of studies. First, viscosity per se does not affect suprathreshold judgments of perceived saltiness and sweetness. The addition of certain gums and starches to taste solutions may affect judgments of taste intensity, but the effect is probably not mediated exclusively or significantly by changes in solution viscosity. Second, an interaction effect is observed when the addition of a thickening agent does affect perceived taste intensity; at high-viscosity levels, judgments of the intensity of only the weak-tasting solutions were significantly lowered. In this series of studies, the addition of CMC-H produced significant reductions in the perceived taste intensities of as high a series of solution concentrations as .12 $\mathrm{M}$ sucrose and .10 M $\mathrm{NaCl}$.

The finding that increasing solution viscosity does not necessarily influence perceived taste intensity receives corroboration from the study by Pangborn et al. (1973). Even when the viscosity range is extended well beyond the range used by these investigators (up to $72 \mathrm{cps}$ ), the same principle emerges. Whether perceived taste intensity is affected by the presence of a thickening agent depends upon the particular gum or starch.

In the present studies, a wide range of viscosity levels and taste concentrations were used with a scaling procedure that permitted sequential, rather than simultaneous, comparisons between test stimuli. A narrower range of sensory levels for test stimuli and the use of simultaneous comparisons should maximize the opportunity to observe smaller, but significant, sensory differences between stimuli (Poulton, 1968; Sandusky, 1974). However, the use of magnitude estimation procedures and a broad range of sensory stimuli may be more representative of the "real world," where a wide variety of stimuli are encountered in a setting that rarely permits simultaneous comparisons with similar stimuli. 
The results of this set of experiments permit only speculation about the mechanism(s) responsible for the selective effects of different types of CMC on taste perception. The three forms of CMC used in this study differ only in polymer length. Perhaps polymer length differentially affects the rate of movement of taste molecules in the viscous matrix. Evidence exists that taste solution flow rates can affect judgments of taste intensity (Meiselman \& Halpern, 1973). It is also possible that taste molecules become differentially bound to the three types of $\mathrm{CMC}$ or that polymer length influences whether $\mathrm{CMC}$ will act as a surfactant at the taste receptor surface and diminish the ability of the receptor cell to recognize taste stimuli.

The concentration of CMC may have contributed to the observed perceptual effects because of the addition of sodium (sodium carboxymethylcellulose). Although subjects reported no salty or sweet tastes associated with the addition of CMC to deionized water alone, the postulated taste-enhancing property of sodium may have reversed a depression in perceived taste intensity which would otherwise have been observed with increasing solution viscosity. Approximately three times more CMC-L than $\mathrm{CMC}-\mathrm{H}$ was required to thicken aqueous solutions to prescribed viscosity levels. As illustrated in Figure 3, with the highest concentrations of CMC (CMC-L at 1,296 and $2,025 \mathrm{csk}$ ), subjects actually perceived the thicker $\mathrm{NaCl}$ solutions to be saltier than the thinner solutions. This trend did not reach statistical significance, but in other research (Pangborn et al., 1973) the addition of CMC-L produced a significant increase in the perceived saltiness of $\mathrm{NaCl}$ solutions and the perceived sweetness of $\mathrm{Na}$ saccharin solutions.

\section{REFERENCES}

Arabie, P., \& Moskowitz, H. R. The effects of viscosity upon perceived sweetness. Perception \& Psychophysics, 1971, 9, 410-412.

Cellulose gum: Sodium carboxymethylcellulose chemical and physical properties. Wilmington, Del: Hercules, Inc., 1971.

Christensen, C. M. Effects of taste quality and intensity on oral perception of viscosity. Perception \& Psychophysics, 1980, 28, 315-320.

ENGEn, T. Psychophysics: II. Scaling methods. In J. W. Kling \& L. S. Riggs (Eds.), Woodworth and Schlosberg's Experimental psychology. New York: Holt, Rinehart \& Winston, 1971.

Meiselman, H. L., \& Halpern, B. P. Enhancement of taste intensity through puisatile stimulation. Physiology \& Behavior, 1973, 11, 713-716.

Moskowitz, H. R., \& Arabie, P. Taste intensity as a function of stimulus concentration and solvent viscosity. Journal of Texture Studies, 1970, 1, 502-510.

Pangborn, R. M., \& Szczesniak, A. S. Effect of hydrocolloids and viscosity on flavor and odor intensities of aromatic flavor compounds. Journal of Texture Studies, 1974, 4, 467-482.

Pangborn, R. M., Thabue, I. M., \& Szczesniak, A. S. Effect of hydrocolloids on oral viscosity and basic taste intensities. Journal of Texture Studies, 1973, 4, 224-241.

Poulton, E. C. The new psychophysics: Six models for magnitude estimation. Psychological Bulletin, 1968, 69, 1-19.

SAndusky, A. Memory processes and judgment. In E. C. Carterette \& M. P. Friedman (Eds.), Handbook of perception (Vol. 2). Psychophysical judgment and measurement. New York: Academic Press, 1974.

Shama, F., \& Sherman, P. Identification of stimuli controlling the sensory evaluation of viscosity: II. Oral methods. Journal of Texture Studies, 1973, 4, 111-118.

STtunens, S. S. In G. Stevens (Ed.), Psychophysics. New York: Wiley, 1975.

Winer, B. J. Statistical principles in experimental design. New York: McGraw-Hill, 1971.

(Received for publication March 12, 1980; revision accepted July 16,1980 .) 